\title{
Numerical analysis of JET discharges with the European Transport Simulator.
}

D. Kalupin $^{[2,3]}$, I. Ivanova-Stanik ${ }^{[4]}$, I. Voitsekhovitch ${ }^{[1]}$, J. Ferreira ${ }^{[5]}$, D. Coster ${ }^{[7]}$, L.L. Alves $^{[5]}$, Th. Aniel ${ }^{[6]}$, J. F Artaud ${ }^{[6]}$, V. Basiuk ${ }^{[6]}$, J. P.S. Bizarro ${ }^{[5]}$, R. Coelho ${ }^{[5]}$, A.Czarnecka $^{[4]}$, Ph. Huynh ${ }^{[5]}$, A. Figueiredo ${ }^{[5]}$, J. Garcia ${ }^{[6]}$, L. Garzotti ${ }^{[1]}$, F. Imbeaux ${ }^{[6]}$, F. Köchl ${ }^{[8]}$, M.F. Nave ${ }^{[5]}$, G. Pereverzevt, O. Sauter ${ }^{[9]}$, B. D. Scott ${ }^{[7]}$, R. Stankiewicz ${ }^{[4]}$, P. Strand ${ }^{[10]}$, ITM-TF contributors ${ }^{*}$ and JET-EFDA Contributors ${ }^{* *}$

[1] EURATOM/CCFE Fusion Association, Culham Science Centre, Abingdon OX14 3DB UK

[2] EFDA-CSU Garching, Boltzmannstr. 2, D-85748, Garching, Germany

[3] Institute of Energy and Climate Research - Plasma Physics, Forschungszentrum Jülich, EURATOM Association, Trilateral Euregio Cluster, D-52425 Jülich, Germany, www.fzjuelich.de/iek/iek-4

[4] Institute of Plasma Physics and Laser Microfusion, EURATOM Association,00-908 Warsaw, Poland

[5] Associação EURATOM/IST, Instituto de Plasmas e Fusão Nuclear, Instituto Superior Técnico, Universidade Técnica de Lisboa 1049-001 Lisboa, Portugal

[6] CEA, IRFM, F-13108 Saint-Paul-lez-Durance, France.

[7] Max-Planck-Institut für Plasmaphysik, EURATOM-IPP Association, Garching, Germany

[8] Association EURATOM-ÖAW/ATI, Atominstitut, TU Wien, 1020 Vienna, Austria

[9] Ecole Polytechnique Fédérale de Lausanne (EPFL), Centre de Recherches en Physique des Plasmas, Association Euratom-Confédération Suisse, CH-1015 Lausanne, Switzerland

[10] Department of Earth and Space Sciences, Chalmers University of Technology, Euratom-VR Association, SE-352 96 Göteborg, Sweden

e-mail of first author: denis.kalupin@efda.org

\section{Abstract}

The "European Transport Simulator" (ETS) [1,2] is the new modular package for 1-D discharge evolution developed within the EFDA Integrated Tokamak Modelling (ITM) Task Force. It consists of precompiled physics modules combined into a workflow through standardized input/output data-structures. Ultimately, the ETS will allow for an entire discharge simulation from the start up until the current termination phase, including controllers and sub-systems. The paper presents the current status of the ETS towards this ultimate goal. It discusses the design of the workflow, the validation and verification of its components on the example of impurity solver and demonstrates a proof-of-principles coupling of a local gyrofluid model for turbulent transport to the ETS. It also presents first results on the application of the ETS to JET tokamak discharges with ITER like wall. It studies the correlations of the radiation from impurity to the choice of the sources and transport coefficients.

\section{ETS transport solver and workflow design}

The ETS adopts a modular approach, where standalone precompiled physics modules ('actors' in the context of the workflow) are coupled into the workflow through standardised interfaces linked with the ITM data-structure. In view of allowing collective development of

\footnotetext{
* See the Appendix to the paper of G. Falchetto et al., Nucl Fus. Submitted

** See the Appendix of F. Romanelli et al., Proceedings of the 24th IAEA Fusion Energy Conference 2012, San Diego, US.
} 
various applications, a general-purpose Workflow Engine, KEPLER ${ }^{[3]}$, was chosen by the ITM-TF. An actor under KEPLER is a modular physics component that solves a given type of physics problem, e.g. equilibrium reconstruction, computation of transport coefficients or heating profiles. The main advantage of a high level of modularity of ITM developed tools is the possibility to optimise the workflow configuration and the choice of physics modules exactly for the modelling aims, degree of sophistication required and computation time.

The transport solver in ETS solves 1-D transport equations for poloidal flux, $\Psi$, electron or ion density, $n_{e}, n_{i}$, electron and ion temperature, $T_{e}, T_{i}$, and toroidal velocity, $u_{i, \varphi}$, as a function of the toroidal flux coordinate, $\rho$. All ion quantities are solved independently for unlimited number of ions.

$$
\begin{aligned}
& \sigma_{/ /}\left(\frac{\partial}{\partial}-\frac{\rho \dot{B}_{0}}{2 B_{0}} \cdot \frac{\partial}{\partial \rho}\right) \Psi=\frac{F^{2}}{\mu_{0} B_{0} \rho} \frac{\partial}{\partial \rho}\left[\frac{V^{\prime}}{4 \pi^{2}}\left\langle\left|\frac{\nabla \rho}{R}\right|^{2}\right| \frac{1}{F} \frac{\partial \Psi}{\partial \rho}\right]-\frac{V^{\prime}}{2 \pi \rho}\left(j_{n i, \text { exp }}+j_{n i, i m p} \cdot \Psi\right) \\
& \left(\frac{\partial}{\partial}-\frac{\dot{B}_{0}}{2 B_{0}} \cdot \frac{\partial}{\partial \rho} \rho\right)\left(V^{\prime} n_{e}\right)+\frac{\partial}{\partial \rho} \Gamma_{e}=V^{\prime}\left(S_{e, \exp }-S_{e, i m p} \cdot n_{e}\right) \\
& \left(\frac{\partial}{\partial}-\frac{\dot{B}_{0}}{2 B_{0}} \cdot \frac{\partial}{\partial \rho} \rho\right)\left(V^{\prime} n_{i}\right)+\frac{\partial}{\partial \rho} \Gamma_{i}=V^{\prime}\left(S_{i, \exp }-S_{i, i m p} \cdot n_{i}\right) \\
& \frac{3}{2}\left(\frac{\partial}{\partial}-\frac{\dot{B}_{0}}{2 B_{0}} \cdot \frac{\partial}{\partial \rho} \rho\right)\left(n_{i} T_{i} V^{\frac{5}{3}}\right)+V^{\frac{2}{3}} \frac{\partial}{\partial \rho}\left(q_{i}+T_{i} \gamma_{i}\right)=V^{\frac{5}{3}}\left[Q_{i, \exp }-Q_{i, i m p} \cdot T_{i}+Q_{e i}+Q_{z i}+Q_{\gamma i}\right] \\
& \frac{3}{2}\left(\frac{\partial}{\partial}-\frac{\dot{B}_{0}}{2 B_{0}} \cdot \frac{\partial}{\partial \rho} \rho\right)\left(n_{e} T_{e} V^{\prime^{\frac{5}{3}}}\right)+V^{\frac{2}{3}} \frac{\partial}{\partial \rho}\left(q_{e}+T_{e} \gamma_{e}\right)=V^{\prime^{\frac{5}{3}}}\left[Q_{e, \exp }-Q_{e, \text { imp }} \cdot T_{e}+Q_{i e}-Q_{\gamma i}\right] \\
& \left(\frac{\partial}{\partial}-\frac{\dot{B}_{0}}{2 B_{0}} \cdot \frac{\partial}{\partial \rho} \rho\right)\left(V^{\prime}\langle R\rangle m_{i} n_{i} u_{i, \varphi}\right)+\frac{\partial}{\partial \rho} \Phi_{i}=V^{\prime}\left(U_{i, \varphi, \exp }-U_{i, \varphi, i m p} \cdot u_{i, \varphi}+U_{z i, \varphi}\right)
\end{aligned}
$$

Here $\frac{\partial}{\partial t}$ are the time derivatives and $\frac{\dot{B}_{0}}{2 B_{0}} \cdot \frac{\partial}{\partial \rho} \rho$ denote the adiabatic compression terms, which emerge due to our choice of $\rho=\sqrt{\frac{\phi}{\pi B_{0}}}$ as the main radial coordinate, where $\phi$ is the toroidal flux and $B_{0}$ is the vacuum magnetic field. $F$ is the diamagnetic function, $\mu_{0}$ is the permeability of free space, $V^{\prime}$ is the volume derivative with respect to $\rho$ and $R$ is the distance from the axis of the torus. The fluxes, $\Gamma_{e}, \Gamma_{i}, q_{i}, q_{e}, \Phi_{i}$, in the equations (1.1) are considered to be the total fluxes through the flux surface $\rho=$ const and consist of diffusive and convective parts, e.g. for density $\Gamma_{e}=-D \cdot \frac{\partial n_{e}}{\partial \rho}+V \cdot n_{e}$, whereas the flux surface averaged transport coefficients, $D$ and $V$, for all channels, as well as the parallel conductivity, $\sigma_{/ /}$, are provided by external transport models coupled to the transport solver. 
Variables, $\gamma_{i}=c \Gamma_{i}$ and $\gamma_{e}=c \Gamma_{e}$, denote the convective component of the heat flux for ions and electrons respectively. Different transport mechanisms (models) can require different multipliers (typically, $c=5 / 2$ or $c=3 / 2$ or $c=0$ ) in front of the convective component of the total heat flux. A choice of particular value is related to a representation of the RHS of the energy equation, $Q_{\gamma i}$, that also can be done differently in different transport models. It means that the multiplier in front of the convective flux is model dependent and has to be attributed to a particular transport model rather than to an entire equation. Thus, in addition to transport coefficients, the transport model will is providing the transport solver with $c$ multiplier and the flow-work term in the RHS of equation. The source terms in equations (1.1) are also coming from external physics modules providing explicit, $j_{n i, \exp }, S_{e, \exp }, S_{i, \text { exp }}$, $Q_{i, \exp }, Q_{e, \text { exp }}, U_{i, \varphi, \text { exp }}$, and implicit, $j_{n i, i m p}, S_{e, i m p}, S_{i, i m p}, Q_{i, i m p}, Q_{e, i m p}, U_{i, \varphi, i m p}$, components of the source for each equation separately. The exchange terms between different components $Q_{e i}, Q_{z i}, Q_{i e}, U_{z i, \varphi}$ are due to particle collisions and being computed internally by the transport solver.

The physics part of the solver evaluates transport equations (1.1) adopting the standardised form with nine numerical coefficients, $a(\rho) b(\rho) c(\rho) \ldots$,

$$
\frac{a(\rho) \cdot n(\rho, t)-b(\rho) \cdot n(\rho, t-1)}{h}+\frac{1}{c(\rho)} \frac{\partial}{\partial \rho}\left(-d(\rho) \cdot \frac{\partial n(\rho, t)}{\partial \rho}+e(\rho) \cdot n(\rho, t)\right)=f(\rho)-g(\rho) \cdot n(\rho, t)
$$

and with boundary conditions on inner and outer boundary in the form,

$\left.v\left(\rho_{\text {bnd }}\right) \cdot \frac{\partial n(\rho, t)}{\partial \rho}\right|_{\text {bnd }}+u\left(\rho_{\text {bnd }}\right) \cdot n\left(\rho_{\text {bnd }}, t\right)=w\left(\rho_{\text {bnd }}\right)$

Here $n(\rho, t)$ and $n(\rho, t-1)$ are profile of plasma parameter at the current and previous time steps.

The numerical coefficients depend nonlinearly on the solution and the equilibrium geometric coefficients. To solve the equation the iterative procedures has been adopted. In each time step the linear equation are solved obtained by putting the value of the coefficients calculated using the solution from previous iteration step. In next iteration step the values of the coefficients are recalculated using the new value of the solution. The relaxation procedure can be used in iteration by defining the new solution and/or the coefficients as linear combination of the last and previous values. The linear equation is solved using implicit backward Euler scheme in time coupled with various approximation method of linear operator (finite difference schemes leading to three diagonal matrix or methods used in RITM code ${ }^{[4]}$ ). The possibility of relaxation method in iterations by defining the new coefficients and/or new solution as linear combination of the old and new values is implemented.

This allows for decoupling of the physics and numeric inside the transport solver and gives user the choice to select from several numerical schemes. Thus it is possible to optimize the code performance for the particular physics problem. Inputs to 1-D transport equations, such as the geometry (2-D equilibrium), the transport coefficients and the sources are provided by standalone modules coupled in a self-consistent way to the transport solver through generalized data structures. 


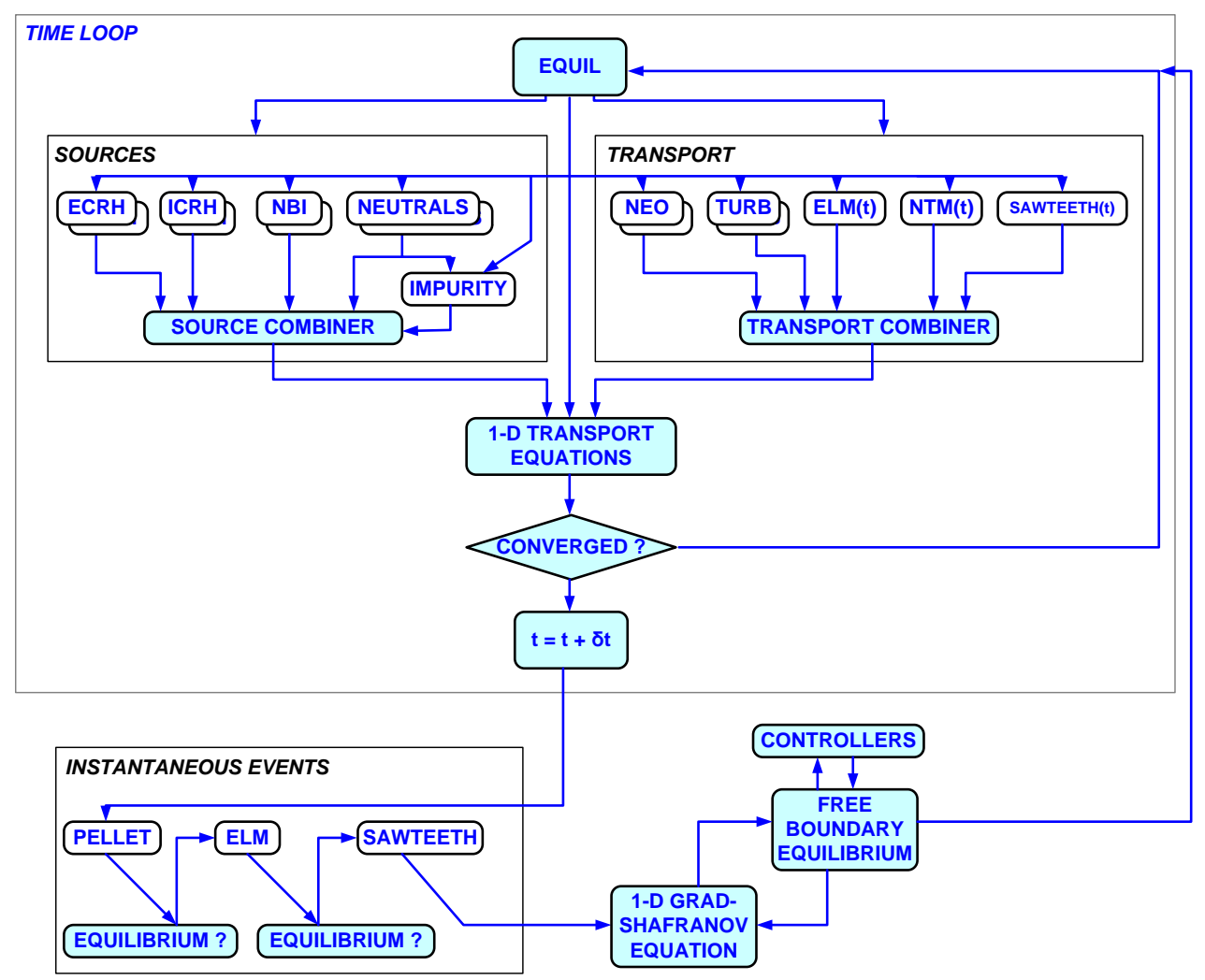

Fig.1: European Transport Solver: a schema of the workflow

A schematic design of the workflow is shown in fig.1. Each box represents a set of modules that treat the same physics problem with various degrees of sophistication. A large choice of equilibrium solvers is available (BDSEQ, EMEQ ${ }^{[5]}, \operatorname{SPIDER}^{[6]}, \mathrm{EQUAL}^{[7]}$, HELENA ${ }^{[8]}$, $\mathrm{CHEASE}^{[9]}$ ). Transport coefficients can be used, provided by neoclassical transport (NCLASS $^{[10]}$, NEOWES, NEOS ${ }^{[11]}$ ) as well as anomalous transport modules of different complexity, from an analytical description (Bohm-GyroBohm ${ }^{[12]}$, Coppi-Tang ${ }^{[13]}$, ETAIGB), to a quasi-linear description $\left(\mathrm{GLF} 23^{[14]}\right.$ or Weiland model $\left.{ }^{[15]}\right)$, up to first-principle based electromagnetic turbulence models (GEM code ${ }^{[16]}$ ) run in parallel on the HPC-FF, a massively parallel supercomputer at Forschungszentrum Julich, as an integral part of the transport simulations. Sources and sinks include the contribution of electron cyclotron heating (GRAY code ${ }^{[17]}$ ), neutral beam injection (NEMO code ${ }^{[18]}$ ), radiation from impurities and Bremsstrahlung radiation, gas puffing, pellet injection and Ohmic power. The total transport coefficients or sources for each equation can also be taken from the database or can be derived as linear combination of values provided by different individual modules. The effect of non-linear MHD modes is taken into account through neoclassical tearing mode or sawteeth modules.

The time stepping is done by the workflow based on the convergence of main plasma profiles. After the entering a new time step the workflow sequentially updates the equilibrium, transport coefficients and sources, then it solves the transport equations.

\section{Verification and Benchmarking of ETS impurity module}

A substantial part of present ITM-TF activities is dedicated to the verification and validation of the developed tools and integrated workflows ${ }^{[19]}$. This is done by means of 
comparison with analytical results using the method of manufactured solutions ${ }^{[1,2]}$, by selfbenchmarking (reduction tests) ${ }^{[1,2]}$, by benchmarking of modules describing the same kind of process against each-other within the same workflow (cross benchmarking) and by benchmarking of the entire workflow against existing transport codes.

The ETS was successfully benchmarked against ASTRA ${ }^{[5]}$ and CRONOS $^{[20]}$ integrated modelling codes.

Before a module is released for productive runs within ETS workflows, it goes through the verification exercises mentioned above. A demonstration is the benchmarking of the impurity module included in ETS.

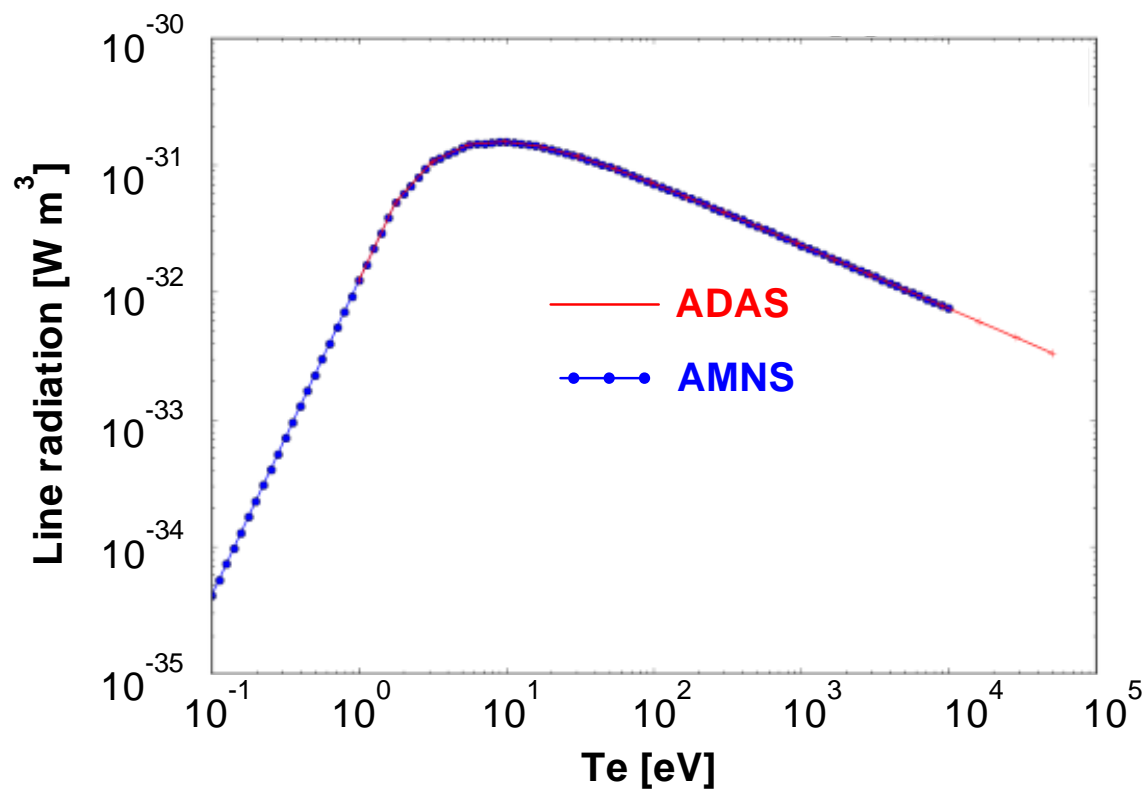

Fig. 2. A comparison of the rate coefficient for line radiation for neutral tungsten from the AMNS system with the data from ADAS, for a density of $1 \times 10^{21}\left[\mathrm{~m}^{-3}\right]$ as a function of electron temperature.

The impurity module in the ETS workflow enables the simulation of the time evolution of the impurity density for an arbitrary number of impurities for all ionisation states ${ }^{[21]}$. The transport coefficients for impurity ions are assumed to be equal to the transport coefficients for deuterium ${ }^{[12]}$. Density sources for each ionization state include ionization, recombination and charge-exchange, where rate coefficients of the relevant process are obtained from $\mathrm{ADAS}^{[22]}$ data base using generic interfaces developed by the ITM-TF. Figure 2 compares the neutral tungsten rate coefficients for line radiation obtained from the ADAS data base directly with the one obtained through the ITM-TF atomic molecular neutral and surface data (AMNS) modules. The perfect agreement between the two curves means that the possible uncertainty introduced by AMNS fitting routines is unlikely to be higher than uncertainty in the original data provided by ADAS consortium.

The impurity solver used within the ETS was benchmarked against the SANCO impurity code, installed as a part of JET analysis suite of codes ${ }^{[23]}$, for conditions of a low confinement mode discharge \#71827 in JET. Tungsten impurity (all ionization states) has been considered. Parabolic profiles for density and temperature of main ions and initial equilibrium provided by EFIT equilibrium reconstruction code were introduced into both codes. Boundary conditions for individual impurity ionisation states were given by the total impurity concentration at the last closed magnetic surface, assuming a coronal distribution at 
the corresponding ion temperature. Figure 3 compares steady state profiles of tungsten ionization states $\mathrm{W}^{31+}-\mathrm{W}^{35+}$ dominating the radiative losses under considered conditions, radiative power density and ion effective ion charge, obtained with ETS and with SANCO after 1s. of time evolution. The obtained total power radiated inside the separaratrix is nearly the same in both simulations. The small discrepancy in profiles of impurity concentration and radiated power density can be explained by 3\% difference in the volume obtained from equilibrium solvers used in compared codes.
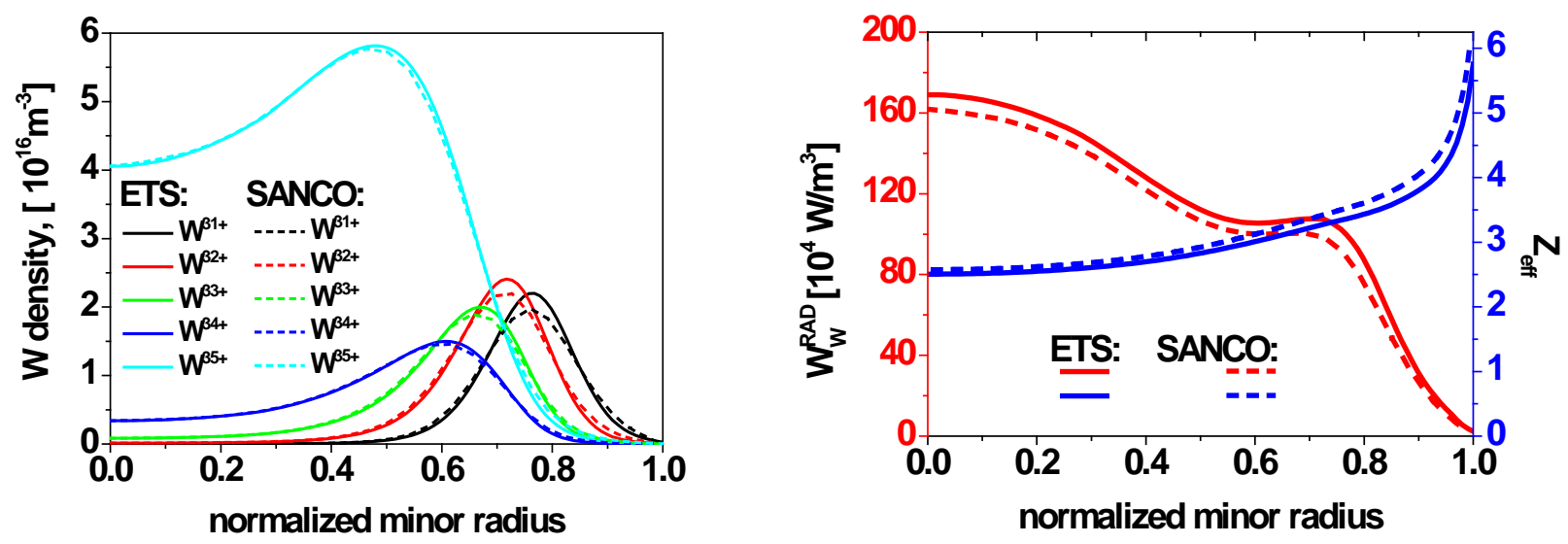

Fig.3 Benchmarking of ETS impurity solver with SANCO code for conditions of JET tokamak assuming parabolic plasma profiles

\section{Physics application results}

\subsection{Impurity Modelling}

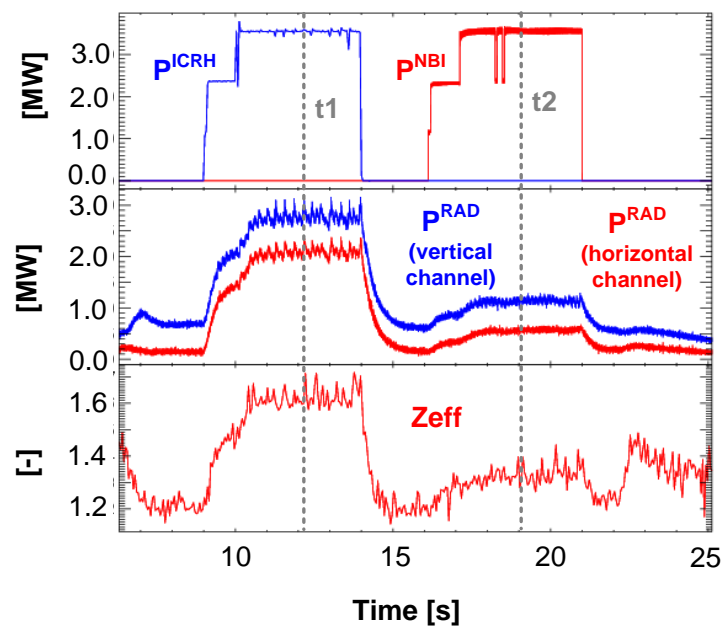

Fig. 4: JET shot \#81856. Experimental time traces of auxiliary heating power, radiative losses and line averaged effective charge). 

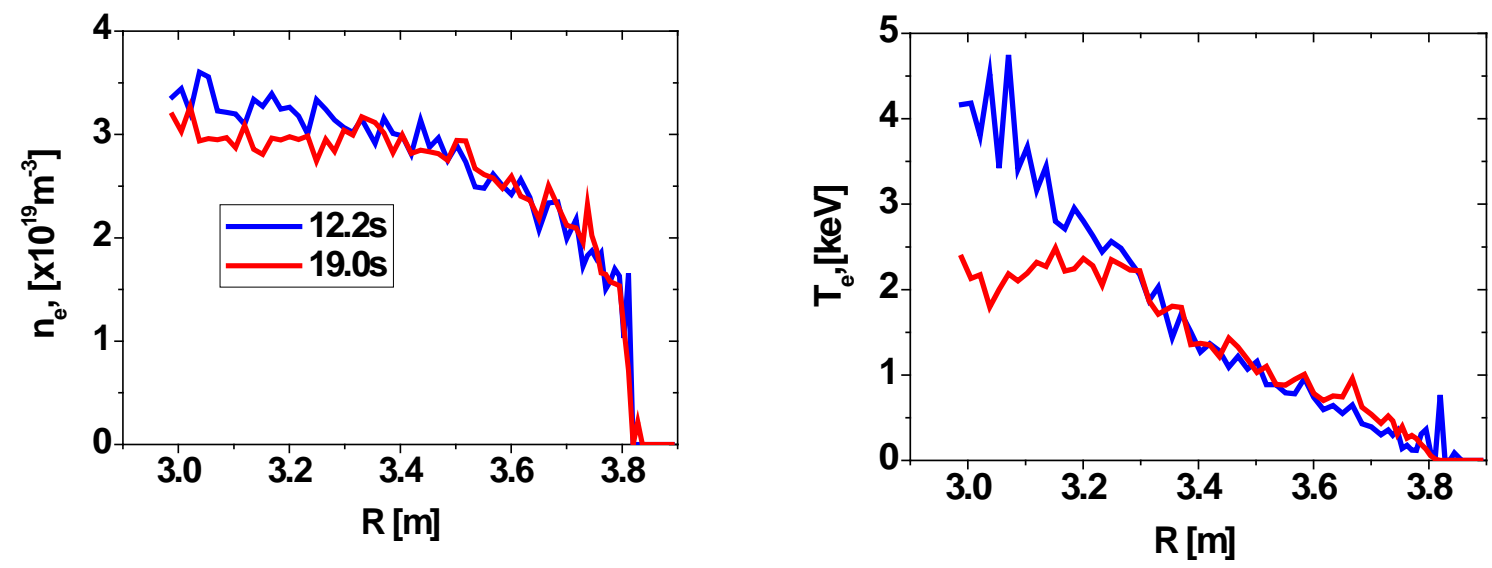

Fig. 5: Comparison of experimental profiles of electron density and temperature for ICRH (blue) and NBI (red) heating phases in discharge \#81856.

The ETS was applied to simulate impurity transport for the conditions of JET discharge \#81856 (ITER like wall) with two phases of 3.5 MW of auxiliary heating delivered by ICRH and NBI respectively (fig. 4) ${ }^{[24]}$. The ICRH results in a substantial increase of both, effective charge, $Z_{e f f}$, and radiative power, $P^{R A D}$, compared to the NBI phase. Main plasma profiles are less affected by the choice of auxiliary heating. Figure 5 compares experimental profiles of the electron temperature and density in both phases. Some difference in temperature is observed within 0.3 of normalized minor radius, which roughly corresponds to $10 \%$ of the volume and can not explain experimentally observed difference in total radiative loss. Thus, the plasma contamination during the ICRH phase can be caused either by an increased source of impurities or by changes in their transport.

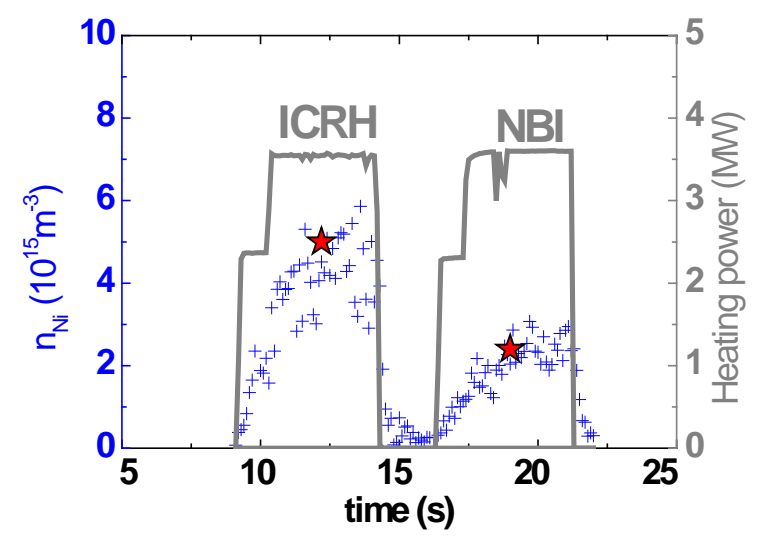

Fig.6 ETS input: Total density of Ni in the discharge \#81856 obtained with SPRED diagnostic (blue crosses) and assumed in ETS simulations (red stars). 


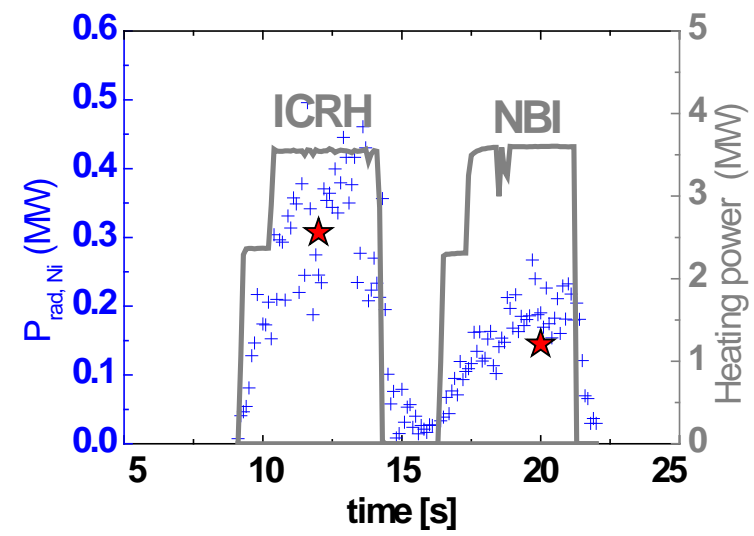

Fig.7 ETS output: Benchmark of radiation (red stars) from Ni obtained with ETS to the one computed from experimental data using UTC code (blue crosses).

Spectroscopic measurements of Ni impurity along the vessel midplane were obtained using the SPRED (survey poor resolution extended domain) spectrometer with the routinely used $450 \mathrm{gmm}^{-1}$ holographic grating. This registers the VUV spectra in the wavelength range 100-1100 A. With the Be/W plasma facing components (PFC) the VUV spectrum was dominated by mid-Z metallic impurities like $\mathrm{Ni}$ and also contained intense $\mathrm{W}$ features. The determination of $\mathrm{Ni}$ impurity densities, based on the combination of absolutely calibrated VUV line transition intensity measurements with the Universal Transport Code (UTC) simulations is described in details in ${ }^{[25]}$. From the Ni density the radiated power due to $\mathrm{Ni}$ and its contribution to the bulk plasma radiated power $\left(\mathrm{P}_{\text {rad,bulk }}\right)$ was evaluated based on calculations of the $\mathrm{Ni}$ cooling factor presented in ${ }^{[26]}$. The time trace of the total density of $\mathrm{Ni}$ obtained with SPRED for the shot \#81856 is shown in fig.6. The Ni concentration is up to the factor of three higher for the ICRH phase.

ETS simulations were configured to reproduce experimental profiles of radiated power density and a value of line-averaged effective charge at selected times (ICRH phase, $t_{1}=12.2$ $\mathrm{s}$; and NBI phase, $\mathrm{t}_{2}=19 \mathrm{~s}$ ). Three impurity species (all ionization states of $\mathrm{W}, \mathrm{Ni}$ and $\mathrm{Be}$ ) have been simulated until the steady state impurity distribution is obtained. Initial profiles and boundary conditions for individual ionization states of impurity ions are obtained from coronal distribution using experimental $n_{e}$ and $T_{e}$ profiles and adjusting total concentration for each impurity. Total Ni concentration was taken from experiment, total $\mathrm{W}$ concentration was adjusted to reproduce radiative losses and Be concentration was adjusted to reproduce $Z_{\text {eff. }}$ For the NBI phase the total boundary concentrations were selected to $n_{W}=1.1 \times 10^{15} \mathrm{~m}^{-3}$, $\mathrm{n}_{\mathrm{Be}}=1.0 \times 10^{17} \mathrm{~m}^{-3}, \mathrm{n}_{\mathrm{Ni}}=2.4 \times 10^{15} \mathrm{~m}^{-3}$, and for the ICRH phase these values were increased to $\mathrm{n}_{\mathrm{W}}=4.5 \times 10^{15} \mathrm{~m}^{-3}, \mathrm{n}_{\mathrm{Be}}=2.2 \times 10^{17} \mathrm{~m}^{-3}, \mathrm{n}_{\mathrm{Ni}}=5.0 \times 10^{15} \mathrm{~m}^{-3}$. The impurity diffusion coefficients have been computed with the L-mode particle Bohm-gyroBohm transport mode ${ }^{[12]}$ and assumed to be equal for all impurity ions. Since the electron temperature and density in the NBI and ICRH phases are similar, computed profiles of transport coefficients are nearly the same. Figure 7 compares the radiated power from Ni obtained in ETS simulation to the one computed directly from experimental data using UTC code. 

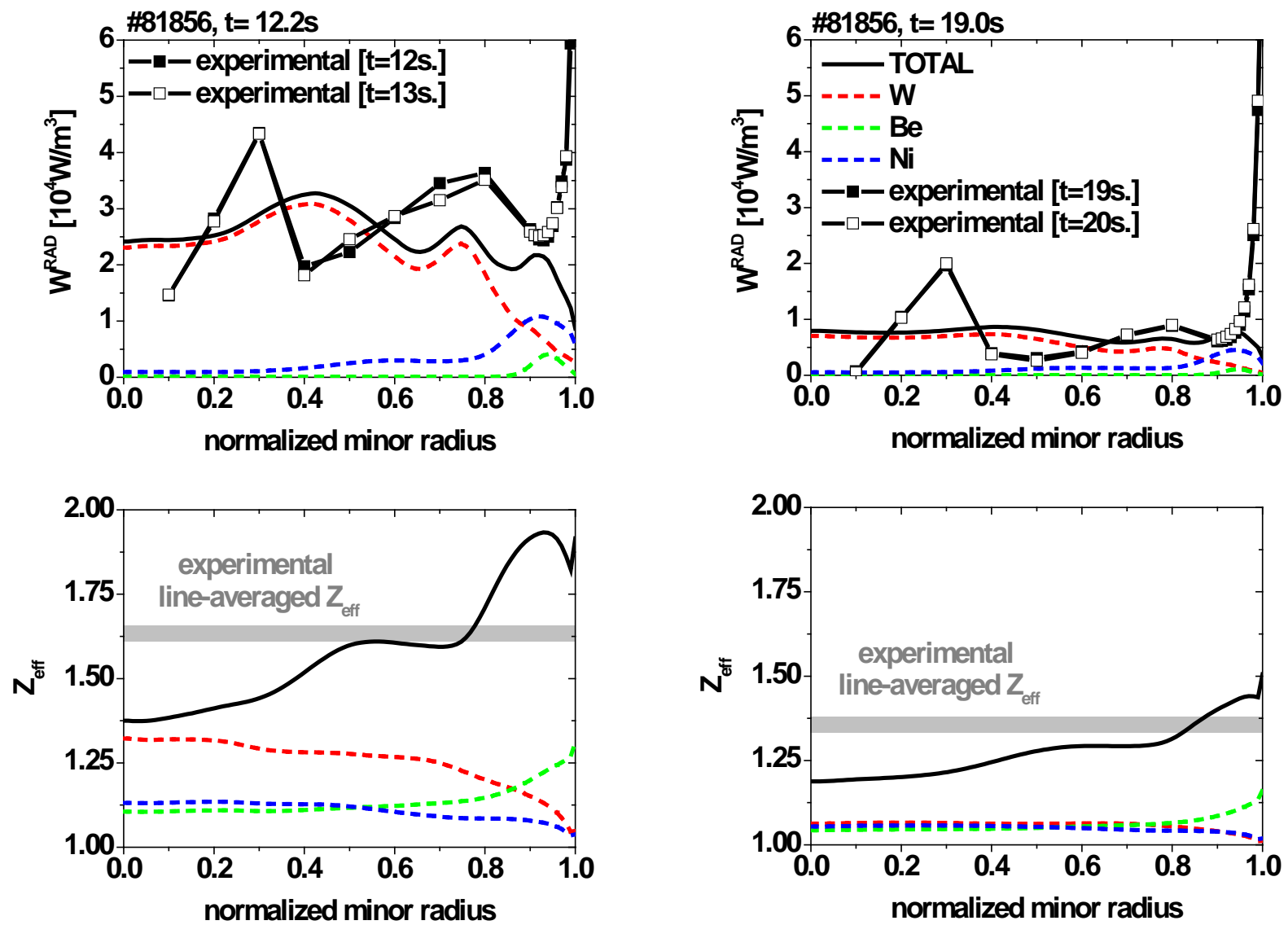

Fig.8 Comparison of simulated steady state profiles of radiative power density and effective charge with experimentally measured ones: black curves - total profiles, red curves - $W$ contribution to the total; blue curves - Ni contribution to the total; green curves - Be contribution to the total.

Figure 8 compares the simulated steady state profiles of radiative power density, $W^{R A D}$, and effective charge with experimentally measured ones. A reasonable agreement for total radiative power density profile inside normalised radius 0.95 has been obtained for both heating phases. The core radiation is dominated by W during both, ICRH and NBI phases, with the largest contribution from $\mathrm{W}^{25}-\mathrm{W}^{35}$ ionization states. The edge radiation is dominated by $\mathrm{Ni}$ ions, whereas Be does not contribute much to the radiative losses. Instead, plasma effective charge is mostly due to light ions, eg. Be and Ni. On the contrary to the radiated power profile, flat or slightly peaked in the centre, the effective charge profile is hollow with the maximum at the edge. These computations exclude light- $\mathrm{Z}$ impurity (like $\mathrm{C}, \mathrm{O}$ or $\mathrm{N}$ ), which dominate the radiation over last centimetres inside the separatrix. Therefore, there is a difference between experimental and computed profiles of the radiated power density outside the normalised radius of 0.95 . The total radiated power caused by the light elements is about $12-15 \%$. Nonetheless, experimental radiation caused by light impurities is not sensitive to the heating scheme. The increased radiation during the ICRH phase as compared to the NBI is due to increased tungsten concentration in the plasma core.

In the simulation performed above the high radiation power during ICRH phase has been obtained by increasing the boundary density for $\mathrm{W}$. Another approach to increase the $\mathrm{W}$ concentration in the core can be an increase of the inward W convection. Another set of simulations including Be and W was performed to study the sensitivity of Prad and Zeff to 
the boundary concentration versus convective velocity of impurity particles. Starting with the NBI phase, the Be and W sources were adjusted through their boundary values to match the experimentally measured impurity concentration and radiative losses $\left(n_{W}=8.0 \cdot 10^{14} \mathrm{~m}^{-3} ; n_{B e}\right.$ $=3.0 \cdot 10^{17} \mathrm{~m}^{-3}$ ) for Be and $\mathrm{W}$ impurity densities assuming zero impurity convective velocity (Fig. 9, blue curves). Taking these results as a reference the impurity distribution during the ICRH phase has been first simulated by assuming a radially constant inward impurity convective velocity of $0.5 \mathrm{~m} / \mathrm{s}$. This results in an increase of $\mathrm{W}^{\mathrm{RAD}}$ and Zeff, mostly at the magnetic axis, where impurities start to accumulate (Fig. 9, green curves). Such $\mathrm{W}^{\mathrm{RAD}}$ profile appeared to be inconsistent with the bolometric measurements showing a rather flat profile (see fig.8) of radiative power during the ICRH phase. In addition, taking into account a small volume contribution from the plasma centre, the total radiative losses change only within a few percent compared to the factor of 2.5 measured in experiment. At the next step, the reference case has been repeated with zero convective velocity and increased (roughly by factor 3) impurity boundary densities $\left(n_{W}=2.35 \cdot 10^{15} \mathrm{~m}^{-3} ; n_{B e}=9.1 \cdot 10^{17} \mathrm{~m}^{-3}\right)$. In this case a much better agreement with measurements for $\mathrm{W}^{\mathrm{RAD}}$ profile and Zeff is obtained. These simulations indicate that an increased impurity density is a possible reason for the $\mathrm{W}$ accumulation during the ICRH phase of \#81856, although the effect of a radially shaped convective velocity (not tested here) can not be excluded.
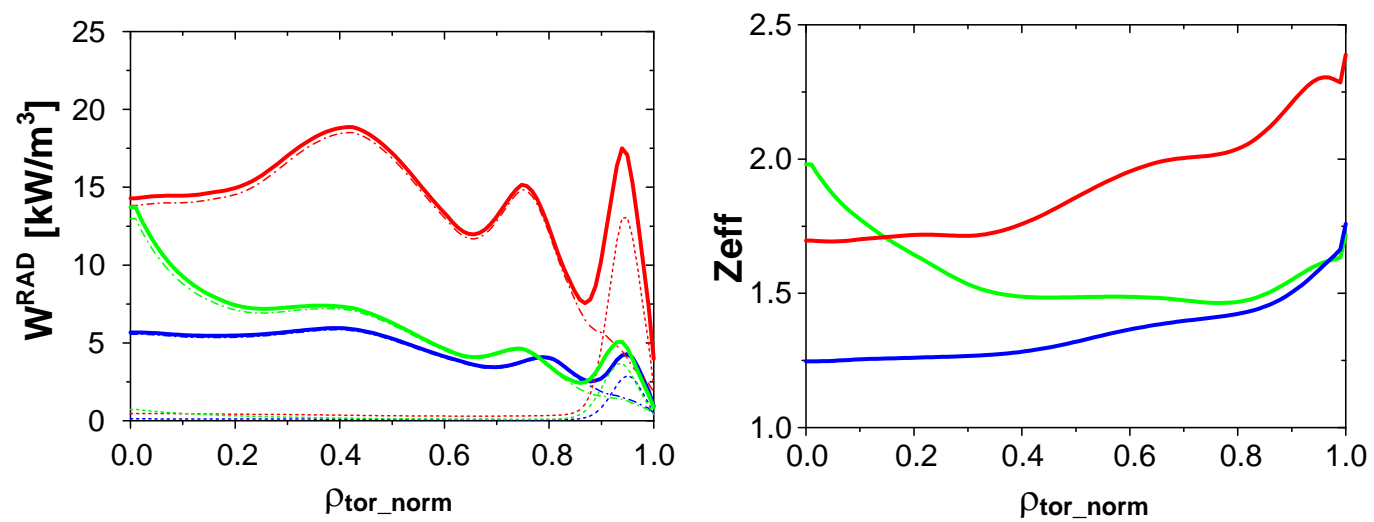

Fig.9 JET shot \#81856. Simulated steady state profiles of radiative power density and Zeff: The reference case (blue curves) corresponds to the NBI phase (time t2). The effects of ICRH

(time t1) are simulated by either increasing the Be and $W$ source (green curves) or increasing the inward Be and W pinch (red curves). Dashed curves - total Be contribution; dash-dot curves - total $W$ contribution to radiative loss.

\subsection{Transport-turbulence coupled simulation}

Another area where the ITM-TF is developing capabilities of the ETS simulator, advanced compared to existing integrated modelling transport codes (e.g CRONOS or JETTO), is the coupling of the core transport solver to turbulence codes. Recently, the electromagnetic gyrofluid turbulence code GEM ${ }^{[16]}$ was implemented in the ETS workflow. It uses the same generic interfaces to transport part as other transport models (like BohmgyroBohm or linear models), providing the ETS with transport coefficiens, $D_{\perp}$ and $V_{\perp}$. GEM is run remotely on HPC-FF as a parallel job while the main part of the workflow is serial and runs on the ITM computing cluster. GEM and ETS use different time and radial scales. GEM is run for the selected number of flux tubes and for each of those receives local gradients and parameters from the transport solver. Once GEM recomputes the fluxes, it turns them into 
transport coefficients which are provided back to the transport solver using linear interpolation between flux tubes. The earlier approaches to couple the gyrofluid and transport codes are presented in ref. ${ }^{[27]}$. The advantage of ITM approach is that GEM can be easily interchanged in the ETS with other turbulence codes, including gyrokinetic codes, once they are adapted to the ITM defined infrastructure.

Figure 10 presents the electron density and temperature profiles time evolution obtained for the conditions of the JET discharge \#71827, starting from the experimental profiles at the time 12s, obtained using GEM calculated transport coefficients for the density and the temperature. GEM is using 256 cores on HPC-FF. It is to be noted that this is a proof of principle, as the run lasted $10 \tau_{G B}$ whereas fully saturated turbulence requires much longer runs. GEM is implemented as a chain of 8 flux tubes, from 0 to 7 , with the i-th case at normalised toroidal flux radius $[(2 \mathrm{i}+1) / 16]^{\wedge} 0.7$. Each flux tube takes parameters from its profile location, runs for 10 gyro-Bohm times, $\tau_{G B}=-\left(c_{S} \cdot d \ln T_{e} / d \rho\right)^{-1}$, and returns transport coefficients. Each case has a different $\tau_{G B}$ with saturation occurring on a scale of about $100 \cdot \tau_{G B}$. The updated transport coefficients are used by ETS on its own (transport) time scale. The time within GEM is used merely to control evolving saturation. A fully relaxed run under ETS should take between 100 and 1000 loop steps, depending on proximity to instability thresholds and pathologies which can occur there. Obtaining fully saturated runs is work in progress. Nevertheless, the general behaviour of this coupled turbulence-transport cases is already visible in Figure 10: the maximum of the computed heat transport coefficient at the edge is due to the nonlinear processes occurring when $V_{T e} / q R>-\left(c_{S} \cdot d \ln T_{e} / d \rho\right)$, where $V_{T e}$ is the electron thermal velocity. In the core, by contrast, the nonlinear long-wavelength character of edge turbulence is absent, therefore the transport level is much lower.
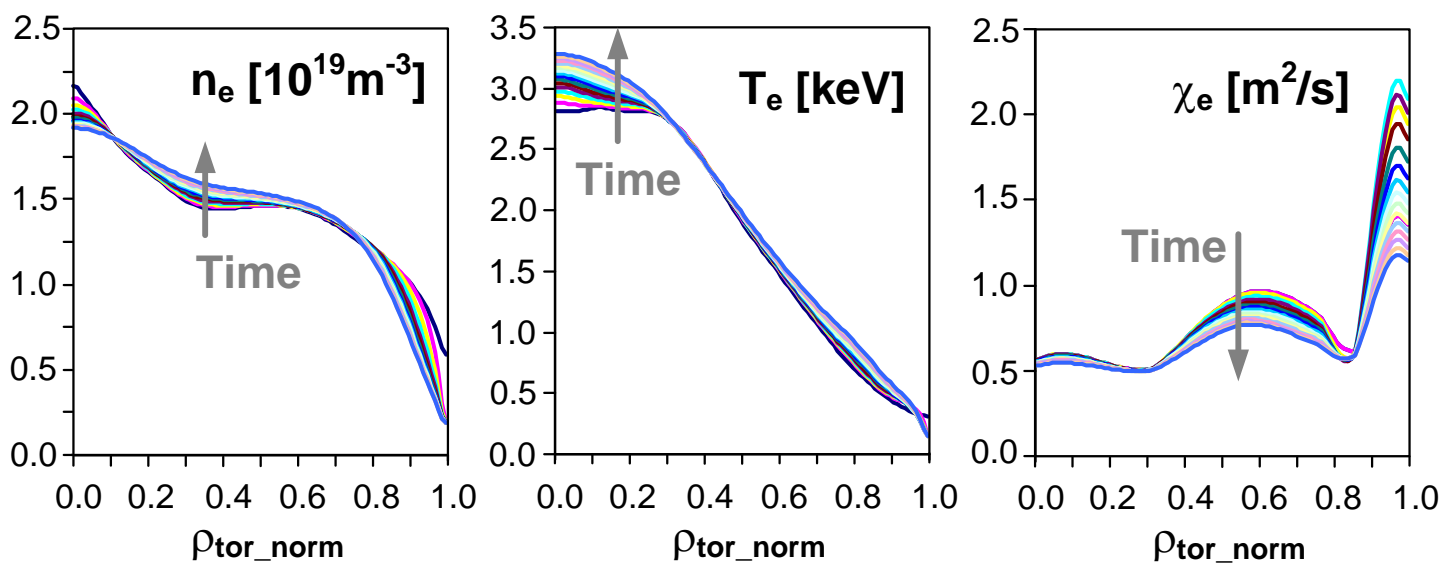

Fig. 10: Transport-turbulence coupled computations: relaxation of electron density and temperature profiles (over 15 transport time steps) due to GEM transport coefficients

\section{Conclusions}

The new modular European Transport Simulator, ETS, developed by the ITM-TF has been applied to simulate the conditions of several JET discharges. The primary objective of these simulations was to verify the modules and demonstrate the functionality of the workflow coupling, including that between the turbulence code and transport solver. The 
ETS workflow was successfully benchmarked against major existing integrated modelling codes ${ }^{[2,28]}$.

Impurity simulations performed for JET discharge 81856 are capable of reproducing the profiles of radiated power and effective charge in a relatively good agreement with the experiment. They show that the increased radiation during the ICRH phase as compared to the NBI phase may be explained by an increased impurity source at the edge. The increase of the radiation is caused mostly by higher concentration of $\mathrm{W}$ in the plasma core, particularly by $\mathrm{W}^{25}-\mathrm{W}^{35}$ ionization states. Instead, plasma effective charge is mostly due to light ions, eg. Be and Ni. On the contrary to the radiated power profile, flat or slightly peaked in the centre, the effective charge profile is hollow with the maximum at the edge. The sensitivity study shows that a possible reason for the $\mathrm{W}$ accumulation during the ICRH phase is an increased concentration of $\mathrm{W}$ at the edge. In simulations it was not possible to achieve a necessary increase of the $\mathrm{W}$ concentration in the centre through the modification of the convective term of impurity transport, although the effect of a radially shaped convective velocity cannot be excluded.

A proof of principle of turbulence-transport coupling was demonstrated with the ETSGEM coupled simulations. The generic behaviour of turbulence driven transport is observed: a relatively moderate transport up to the mid radius is combined with a sharp radial increase of the transport coefficient at the edge due to nonlinear processes.

\section{Acknowledgments}

This work, supported by the European Communities under the contract of Association between EURATOM- CEA, CCFE, IPP, IPPLM, IST, ÖAW, Swiss Confederation, VR, was carried out within the framework of the Task Force on Integrated Tokamak Modelling of the European Fusion Development Agreement. The views and opinions expressed herein do not necessarily reflect those of the European Commission.

\section{References}

[1] Coster D.P., et.al., Plasma Science, IEEE Transactions on, 38(9):2085 -2092, 2010

[2] Kalupin D., et al., In Europhysics Conference Abstracts (Proc. of the 38th EPS Conf. on Plasma Physics, Strasbourg France, 2011) P. 4.111, Vol. 35G

[3] https://kepler-project.org/

[4] Tokar M.Z., Kalupin D, and Pilipenko D., Comput. Phys. Communications 175(1):6 (2006)

[5] Pereverzev, G.V., Yushmanov, P.N., "ASTRA - Automated System for Transport Analysis in a Tokamak", Report IPP 5/98 (2002).

[6] Ivanov A.A. et al., 32nd EPS Conf. on Plasma Phys., ECA Vol.29C, P-5.063 (2005)

[7] Zwingmann W., et al., 2008, PLASMA 2007, AIP Conf. Proc. Vol. 993, 11.

[8] Huysmans G., Goedbloed J., and Kerner W., 1991, in CP90 Conf. on Comp. Physics Proc, page 371,World Scientific Publ. Co.

[9] Luetjens H., Bondeson A., Sauter O., 1996, Comput. Phys. Commun. 97, 219

[10] Houlberg W.A., et al., Phys. Plasmas 4 (1997) 3230

[11] Sauter O. et al, Physics of Plasmas 9, 5140 (2002)

[12] Garzotti L., Proc. 39th EPS Conference on Plasma Physics 2012, P1.004 
[13] Jardin S.C. et al., TSC simulation of ohmic discharges in TFTR - Nucl. Fusion 1993, v.33 p. 371

[14] Waltz R.E. et al 1997 Phys. Plasmas 72482

[15] J. Weiland, “Collective Modes in Inhomogeneous Plasma”, Institute of Physics Publishing, Bristol, (2000)

[16] B Scott, Phys Plasmas 12 (2005) 102307

[17] Figini L. et al., 2012, EPJ Web of Conferences 32, 01011

[18] Schneider R., et al., 2006, Contrib. Plasma Phys. 46, 3, DOI 10.1002/ctpp.200610001

[19] Kalupin D., et al., In Europhysics Conference Abstracts (Proc. of the 35th EPS Conf. on Plasma Physics, Hersonissos, Crete, 2008), P-5.027, Vol. 32D

[20] Artaud J.F., Basiuk V., Imbeaux F., et al., Nucl. Fusion 50 (2010) 043001

[21] Zagorski R., et al., Contrrib. Plasma Phys. Vol.52, No.5-6, 379-383(2012)

[22] Summers H. P., (2004) The ADAS User Manual, version 2.6 ***

[23] Wiesen S., et al, JET ITC-Report 2008

[24] Mayoral M.-L., Proc. 24 ${ }^{\text {th }}$ IAEA Fusion Energy Conference 2012 [EX/4-3] ${ }^{* * * *}$

[25] Czarnecka A., et. al., Plasma Phys. Control. Fusion 53 (2011) 035009

[26] Post D., et. al., At. Data Nucl. Data Tables 20 (1977) 397

[27] Barnes M., et. al., Physics of Plasmas 17 (2010) 0561109

[28] Falchetto G., et. al., 24 ${ }^{\text {th }}$ IAEA Fusion Energy Conference 2012 [TH/P2-25] ${ }^{* * * *}$

**** http://www.adas.ac.uk

${ }^{* * * * *}$ http://www-naweb.iaea.org/napc/physics/FEC/FEC2012/index.htm 